\title{
Full-field optical coherence tomography
}

\author{
H D Ford and R P Tatam \\ Optical Sensors Group, Centre for Photonics and Optical Engineering, \\ School of Engineering, Cranfield University, Cranfield, Bedford, MK43 0AL, UK.
}

\begin{abstract}
To eliminate mechanical scanning in the probe head of an endoscopic OCT system, we propose the use of an imaging fibre bundle for probe beam delivery. Each fibre in the bundle addresses a Fizeau interferometer formed between the bundle end and the sample, allowing acquisition of information across a plane with a single measurement. Depth scanning components are now contained within a processing interferometer external to a completely passive endoscope probe.

The technique has been evaluated in our laboratory for non-biological samples, including glass/air and mirrored/air interfaces. Images resulting from these experiments are presented. The potential of the system is assessed, with reference to SNR performance and acquisition speed.
\end{abstract}

Keywords: OCT, coherence tomography, imaging bundles, coherent fibre bundles, Fizeau interferometer

\section{INTRODUCTION}

Optical coherence tomography (OCT) is a medical imaging technique employing low-coherence interferometry to image the morphology of biological tissue or non-biological semi-transparent composite materials. Appropriate illumination wavelengths, in the visible and near infra red regions of the spectrum, are strongly scattered by tissue, limiting the maximum imaging depth to about $3 \mathrm{~mm}^{1}$. However, the spatial resolution, in the range of about 1 to $30 \mu \mathrm{m}$, is superior to that achieved by other tomographic methods such as ultrasound, enabling cell-level detail to be observed in the highest resolution systems ${ }^{2}$. Additionally, the use of near infra-red radiation, which is considered clinically safe at moderate powers, is preferable to the ionising radiation required by techniques such as X-ray and PET. OCT is therefore of interest both for in-vitro applications and for in-vivo measurements of epithelial and endothelial tissues ${ }^{3,4}$. In applications such as detection of heat damage or pre-cancerous conditions, OCT has the potential to replace, or at least to reduce the necessity for, invasive screening by excision of tissue samples ${ }^{5}$.

Laboratory testing can be conveniently performed using bulk-optic OCT instrumentation, but the acquisition of in-vivo images, typically from within the gastro-intestinal tract, requires the use of a flexible, endoscopic probe. This is realised by incorporating optical fibre components. In early systems, the typical configuration comprised a Michelson interferometer formed from a directional coupler ${ }^{6}$. However, more recent implementations generally employ balanced detection to increase the signal-to-noise ratio (SNR), and sometimes replace the coupler by an optical circulator, resulting in greater optical efficiency and a still higher SNR?

The majority of bulk and fibre-based OCT systems currently under investigation are based around a Michelson interferometer configuration. This allows an instrument to be constructed using relatively few components and provides good signal-to-noise performance. However, optical fibre versions of the Michelson configuration are susceptible to signal fading caused by bending-induced differences between the states of polarisation (SOP) of light in the reference and signal arms ${ }^{8}$. This is evidently more likely to be a problem during in-vivo measurements. Phase drifting of the fringes, caused by temperature fluctuations, is also likely under these conditions. Previous work in our laboratory has demonstrated that, for fibre-based OCT systems, the Michelson interferometer configuration may not always be the optimum choice. A Fizeau interferometer configuration, incorporating an optical circulator and balanced detection, can offer a SNR close to that of the Michelson version, with the additional advantage of down-lead insensitivity ${ }^{9,10}$. Discussion of the characteristics of our current Fizeau OCT system forms the basis of another paper in this conference ${ }^{11}$.

Nano- and Micro-Metrology, edited by Heidi Ottevaere, Peter DeWolf, Diederik S. Wiersma,

Proceedings of SPIE Vol. 5858 (SPIE, Bellingham, WA, 2005)

0277-786X/05/\$15 - doi: 10.1117/12.612566

Proc. of SPIE 58580J-1 
The usual procedure in OCT is to scan the position of the focused probe beam rapidly across the sample surface to build up a 3D map of tissue structure ${ }^{12}$. In this paper, we discuss the adaptation of the Fizeau configuration for use with a coherent fibre bundle, sometimes known as an imaging bundle, to aquire 3D information with a single depth scan. The many thousand fibres within a coherent bundle are arranged in a regular array throughout the entire length of typically several metres ${ }^{13}$. An image projected onto one polished end of the bundle is thus transmitted unchanged to the opposite end. In the proposed bundle-based OCT system, a separate Fizeau interferometer is formed at the output of each fibre in the bundle, enabling OCT information to be transmitted simultaneously from the measurement region over a 2D region of the sample. Detection of the optical signal over the area addressed by the bundle is now performed by a digital camera array, allowing a 3D image to be constructed from a single depth scan.

\section{PRINCIPLES OF COHERENCE TOMOGRAPHY}

The structure that appears in an OCT tissue image results from refractive index discontinuities at boundaries betweeen different types of tissue. At such a boundary, an optical reflection is generated, much larger in magnitude than the level of backscatter from within the continuum of the tissue. The task of the OCT system is to detect and locate the positions of these boundary reflections with the maximum possible spatial resolution.

Sometimes the source used is a swept laser but, most often, illumination is provided by a broadband non-laser device. The wavelength of choice is around $1300-1550 \mathrm{~nm}$, where absorption of light by the tissue is at its lowest ${ }^{14}$. The output spectrum should be as broad as possible, since depth resolution is determined by the coherence length, which is inversely related to source spectral width. For a typical superluminescent source, the spectral width is about $40-100 \mathrm{~nm}$, resulting in a coherence length (and depth resolution) of 10-20 $\mu \mathrm{m}$. A typical single-spot OCT system consists of a Michelson interferometer in which the reference and sample arms are of closely similar length, as shown in figure 1.

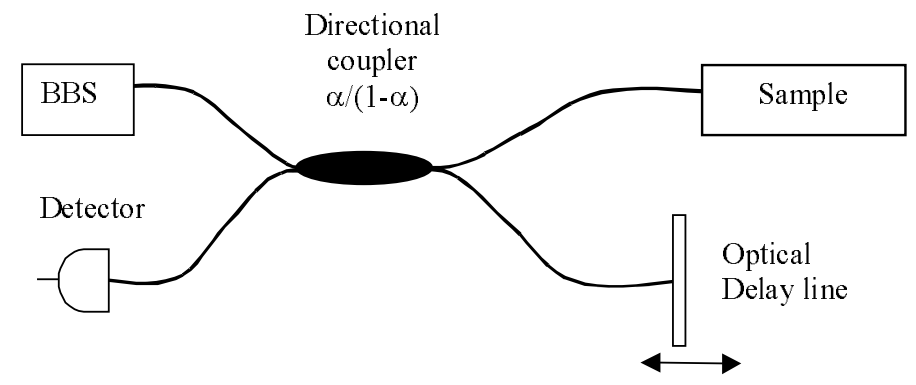

Figure 1. Michelson-configuration OCT system, based on an optical fibre direction coupler. BBS = broad band source, and $\alpha$ is the splitting ratio of the coupler.

The reference arm includes some form of scanned optical delay line, enabling the reference beam path to be swept linearly over a range of several millimetres. For a tissue boundary at a particular depth within the sample, interference fringes will be seen only when the reference beam path is set to match that of the signal beam, to within the 10-20 $\mu \mathrm{m}$ coherence length of the source. When this condition is satisfied, a burst of interference fringes is detected, modulated by the source spectral envelope. Thus the positions of the various tissue boundaries can be located and mapped with high resolution as the reference path is scanned.

Spatial resolution in the plane normal to the optical axis is determined by the spot size of the focused beam within the sample, and is typically similar to the depth resolution. Clearly it is affected by the quality of the focusing optics in the probe head.

The refractive indices of the diverse biological materials within a particular sample of plant or animal tissue are typically rather similar, differing in the third, or at most the second, decimal place. This implies low reflectivity values, and the requirement for appropriate, high SNR detection techniques to allow identification of the interference signals. Balanced detection is widely used to achieve sufficient SNR performance. Imaging depth is ultimately limited to about $3 \mathrm{~mm}$ by decreasing signal strength as light is scattered and absorbed within the sample. 


\section{PROPOSED FIZEAU-CONFIGURATION OCT SYSTEM USING A COHERENT OPTICAL FIBRE BUNDLE}

For single-spot OCT instruments, 3D information is obtained by rapid scanning of the probe beam, not only through the sample depth, but also in a pattern across the sample surface ${ }^{12}$. This works well in many situations, and typically allows a 3D image of a volume up to a few cubic millimetres to be obtained in a few seconds. However, when using this type of system endoscopically, for internal in-vivo measurements, active scanning is required at the endoscope tip. Although this has been successfully demonstrated using miniaturised components ${ }^{15}$, it is not straightforward, and a passive probe would be advantageous for endoscopic use.

A coherent fibre bundle, by allowing simultaneous OCT information to be obtained from an array of positions across the sample, eliminates the requirement for $\mathrm{x}-\mathrm{y}$ scanning. The bundle diameter of a few $\mathrm{mm}$ is ideal for endoscopic use and a fibre spacing as little as $6-10 \mu \mathrm{m}$ can be obtained. The potential spatial resolution is therefore similar to that obtained from an $\mathrm{x}-\mathrm{y}$ scanned system.

The basic proposed configuration for a bundle-based OCT system is shown in figure 2 . It is very similar to the arrangement employed in our laboratory for a single-point system, and is based around a Fizeau-type probe interferometer. This is formed between the end face of the bundle and the sample itself. As described in paper 5858-18, path length matching cannot be achieved directly in this arrangement, so a scanned processing interferometer is employed, having an average path-length mismatch identical to that of the Fizeau. In the work presented here, the processing interferometer is of the Michelson type, but other configurations are possible.

The Fizeau arrangement is particularly appropriate for the bundle-based system, because the bundle itself does not form part of either the sample or the processing interferometer. This is important, for several reasons, the most obvious of which is that the instrument now has 'down-lead' insensitivity. Environmentally-induced phase and polarisation changes within the bundle do not affect the visibility of the interference signal.

\section{EXPERIMENTAL DETAILS}

The experiments described in this paper were performed using the configuration shown in figure 2 . The source was a 5 $\mathrm{mW}, 830 \mathrm{~nm}$ superluminescent diode (SLD), with a bandwidth of about $15 \mathrm{~nm}$, corresponding to a coherence length of about $30 \mu \mathrm{m}$. Although this is not the optimum wavelength region for tissue measurements, it was chosen for these proof-of-principle experiments to fall within the appropriate range for good sensitivity of the CCD camera used as the detector. Light from the source was collimated and transmitted through the processing interferometer to the input face of the imaging bundle, via a beamsplitter cube which allowed $50 \%$ of the returning light to be directed onto the detector. An optional focusing lens (A) enabled the beam diameter to be adjusted to illuminate any required area of the bundle face. Focusing after the processing interferometer is the preferred method, since this interferometer has unequal path lengths in the two arms. If the focusing lens were positioned before the interferometer, a wavefront curvature mismatch would arise, resulting in bulls-eye fringes in the recombining beams.

\subsection{Imaging bundle}

The imaging bundle was $2 \mathrm{~m}$ in length and was made up of 300x300 fibres on a $10 \mu \mathrm{m}$ centre spacing. The light was transmitted by the bundle to a probe head comprising a pair of achromats positioned in a unit magnification, $4 f$ imaging arrangement. Achromat lenses were used to reduce aberrations, since image quality is of critical importance in the bundle-based system. 


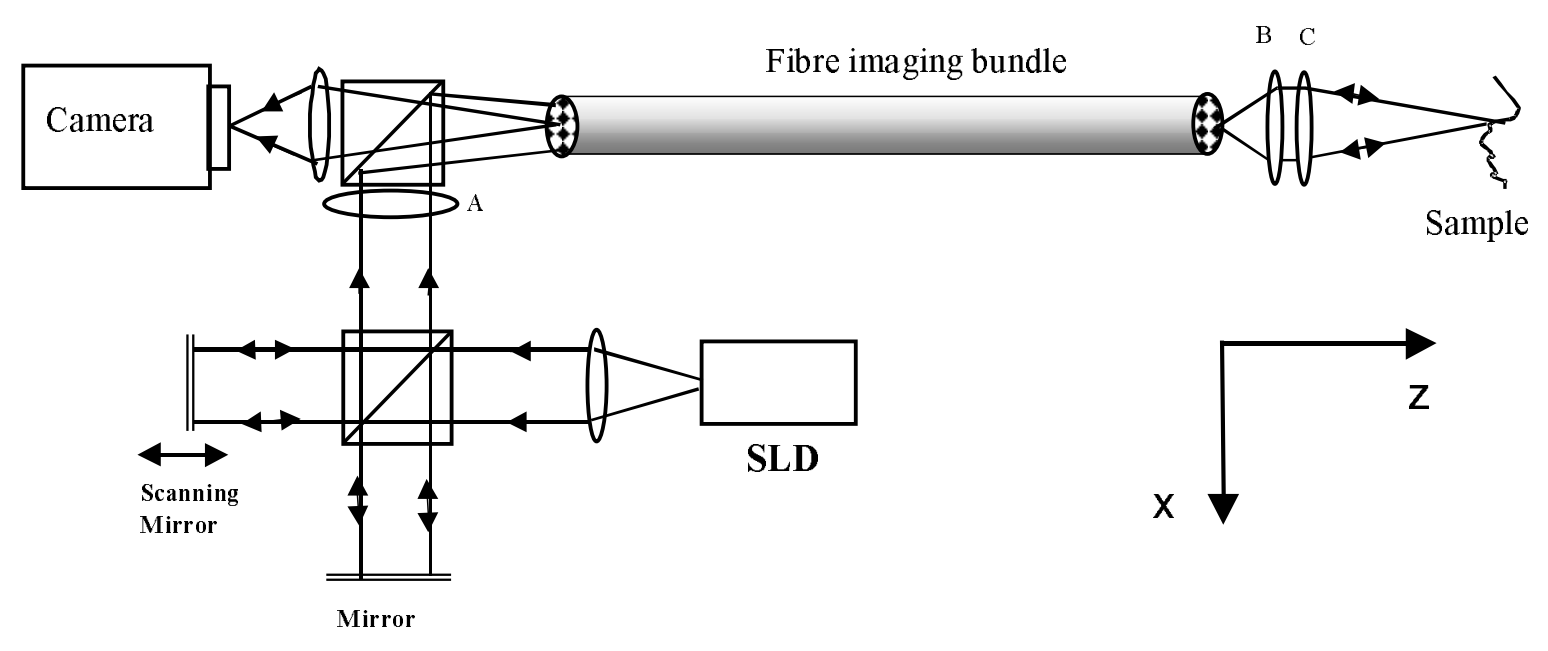

Figure 2. Configuration for 3D OCT employing a fibre imaging bundle. SLD = super-luminescent diode, $\mathrm{A}=$ optional focusing lens controlling illumination onto bundle face, $\mathrm{B}$ and $\mathrm{C}$ are probe head focusing lenses. The co-ordinate system used is as shown in the figure, with the $y$-direction into the plane of the paper.

In a conventional, single point OCT system, the beam waist diameter determines the spatial resolution of the instrument in the x-y plane normal to the beam direction. Poor focusing degrades the spatial resolution. In the bundle-based system, this is also the case. However, there is now an additional consequence of poor focusing. If the beam waist diameter becomes larger than the fibre spacing, cross-talk arises between the fibres in light scattered by the sample. Nonreciprocal imaging can take place, in which light transmitted to the sample by one fibre can be backscattered and collected by a different fibre. Since the path length in the Fizeau varies depending on which pair of neighbouring fibres are involved, spurious sets of interference fringes can arise at the detector. Figure 3(a) shows the intensity variation for four individual fibres from the bundle as the processing interferometer is scanned, when ordinary plano-convex lenses are used in the probe head. Multiple fringe bursts can be seen arising from severe cross-talk. The consequences are blurring of interfaces in the OCT image and degradation of both depth (z) and $x-y$ resolution. Achromats were found to perform significantly better in our probe head, over the small field of view required, than standard plano-convex lenses, although their ability to minimise aberrations decreases rapidly for off-axis object points. With the aperture used, the Rayleigh range was about $300 \mu \mathrm{m}$, limiting the scan depth for acceptable imaging to about twice this distance.

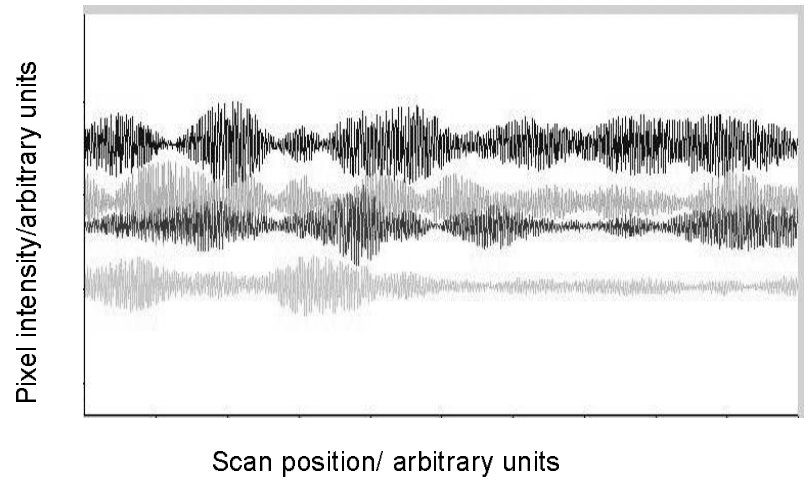

(a)

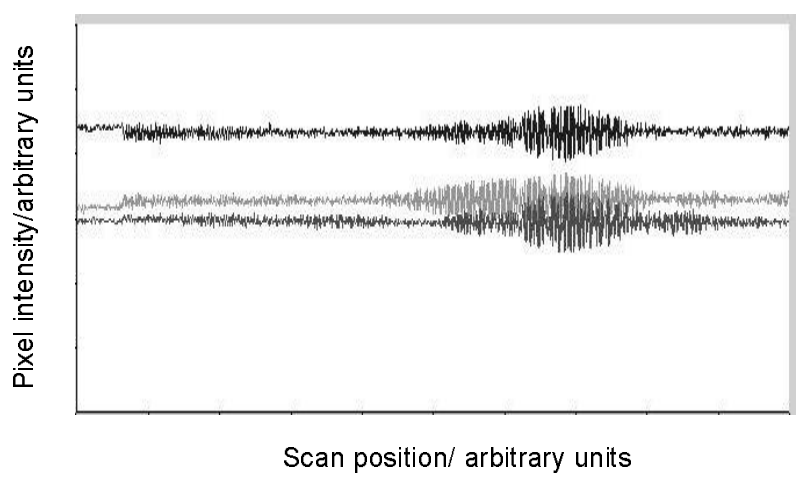

(b)

Figure 3. Intensity modulation of the signal from individual fibres in the imaging bundle as the processing interferometer is scanned, when using (a) standard plano-convex and (b) achromat lenses for focusing in the probe head.

By choosing an appropriate aperture diameter for our system, focusing quality with achromats was sufficient to limit any cross-talk to the immediate neighbours only of a particular fibre. Typically, no more than one additional pair of 
interference envelopes was seen to either side of a true interference signal, and the magnitude of the satellite envelopes was reduced to an almost negligible level. Figure 3(b) shows the intensity modulation for three individual fibres when achromats replaced the standard lenses in the probe head.

The numerical aperture of the fibre bundle is large, having a value of 0.63 . Under coherent illumination from a laser, and viewed at high magnification, it becomes apparent that the fibres in the imaging bundle support numerous spatial modes. This could present a problem if the bundle were to form part of an interferometer arm, but in the arrangement proposed here, the bundle simply acts as an image conduit to transmit light from the measurement region to the detector. To avoid unwanted intensity fluctuations, it is advisable to set the detector pixel-binning to ensure that each compound pixel covers a region of at least half of the area occupied by a single fibre in the camera image. No other difficuties arise as a result of the multi-moded behaviour of the bundle. Similarly, because the bundle is external to the interferometers, any slight variation in the physical length or optical path difference for different regions of the bundle is unimportant.

The fill-factor for the bundle used in this project, in which fibres were arranged on a square grid, was about $30-40 \%$. This is because data is collected only from the fibre core area, with the regions between fibres appearing dark. Use of a bundle therefore gives a somewhat 'speckled' appearance to the images. The spatial resolution, however, is high, and interpolation or slight smoothing can be used to improve the visual appearance of the OCT images.

\subsection{Detection and processing}

The detector was a 12-bit digital camera with a 640 by 480 pixel array and an integration time variable between 1 and $10000 \mu \mathrm{s}$. The input face of the fibre bundle was imaged, via the beamsplitter onto the image plane of the camera, with a magnification of about 4 to 5 . Thus, in the image, a single fibre occupied an area of 16-25 pixels.

OCT data are acquired from the camera by downloading successive frames into image buffers as the mirror in the processing interferometer is scanned over a distance of typically $0.2-2 \mathrm{~mm}$

Processing software for data gathered from the bundle-based OCT system is under development. Currently, the data are processed and displayed as $2 \mathrm{D}$ slices, showing either $\mathrm{x}-\mathrm{z}$ planes (depth slices) or $\mathrm{x}-\mathrm{y}$ contour maps. In x-z mode, the sampling is set to produce data at 300-400 depth positions over a total depth scan range of about $600 \mu \mathrm{m}$, allowing for each fringe envelope encountered to be sampled at least ten times with this source.

In the $x-y$ mode, sample interfaces are identified by applying a sequential subtraction algorithm to pairs of incoming images, and monitoring the difference value obtained for each pixel. When this value reaches a maximum, corresponding to the peak of a fringe envelope, the scan position is recorded in the final output buffer ${ }^{16}$. At present, this software detects only a single maximum value per pixel, but is under modification to allow the positions of multiple local maxima to be stored, allowing full 3D data display.

A number of samples were investigated, composed of combinations of mirrored and glass surfaces in air. The results of these measurements are detailed below.

\section{RESULTS}

The ability of the system to detect surfaces of varying reflectivity was assessed by interposing a $200 \mu \mathrm{m}$ thick microscope cover slip across and in contact with a mirror placed in the sample position, so as to cover about half of the camera image. Figure 4 shows (a) an en-face image, (b) a depth slice image, (c) a representation of the sample and (d) a 3D plot generated from the en-face image with slight smoothing applied using a $3 \times 3$ median filter.

The interface corresponding to the mirror surface can be clearly identified in both representations. The portion of this interface that is in contact with the coverslip appears shifted in the images by about $100 \mu \mathrm{m}$. This is because the cover slip increases the optical path length in the Fizeau by a distance $t\left(n_{g}-n_{a}\right)$, where $t$ is the cover slip thickness and $n_{g}=1.5$ and $n_{a}=1$ the refractive indices of the glass and of air respectively.

The sample area addressed is about $1.4 \mathrm{~mm} \times 1 \mathrm{~mm}$, with a scan depth of about $0.4 \mathrm{~mm}$, and the depth slice corresponds to the central row in the en-face image, as shown.

The glass/air interface at the front of the cover slip produces only a $4 \%$ reflection, and is therefore more difficult to detect than the mirrored surfaces. However, although much fainter than the signals produced from the mirror reflections, it is visible in the depth slice as a second line near to the top of the image. 


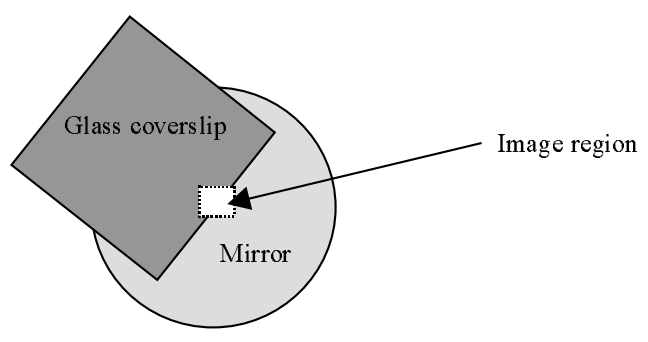

(a)

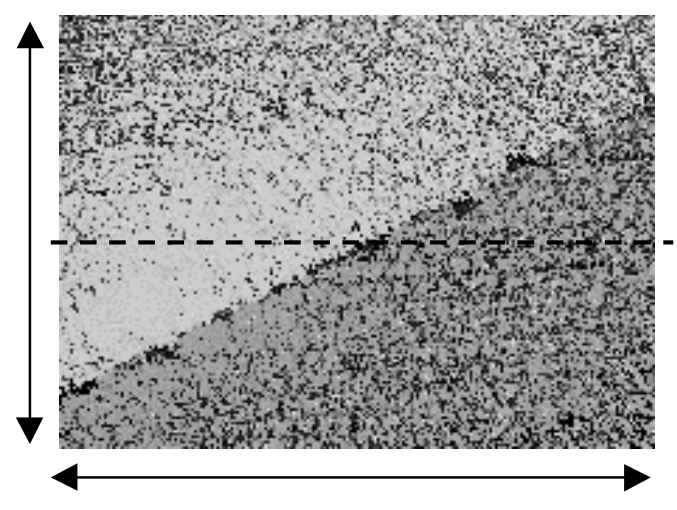

(b)

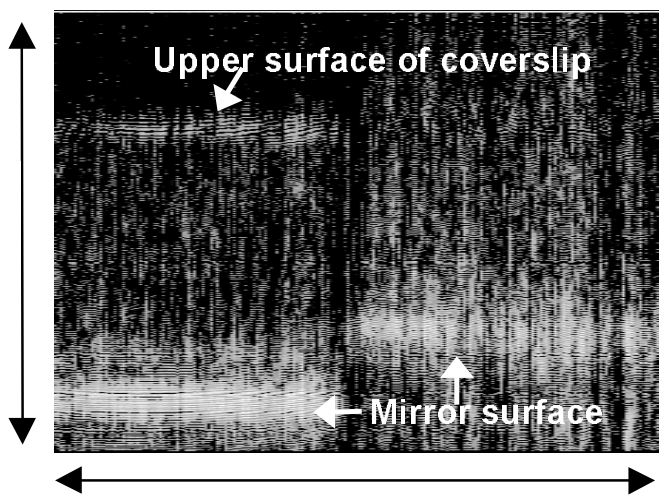

(b)

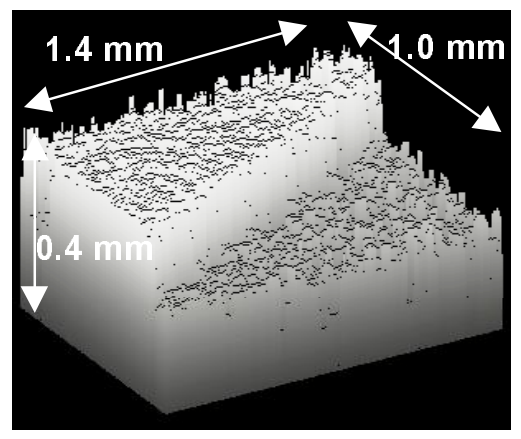

(d)

Figure 4. (a) Representation of the sample composed of a mirror and a glass coverslip. (b) En-face OCT image of sample. (c) Depth slice OCT image corresponding to the position of the dotted line in (b). (d) 3D plot generated from smoothed version of image (a).

The noise level is quite high in figure 4(b). This is probably due primarily to the large dc component arising from the mirror reflection. A second sample was therefore assembled, comprising two stacked glass coverslips mounted on a background of black card. A diagram of this sample, and of a depth slice imaged obtained from it, are shown in figure 5. The en-face processing technique was not, in its current form, appropriate for investigation of this sample, as it would detect for each image position only the interface giving rise to the strongest interference signal, and would ignore other interfaces. Therefore the depth slice image only is shown here.

Four glass/air interfaces can be seen in the left half of figure 5, and two in the right half, as expected, at the appropriate relative positions for the arrangement of cover slips. The two adjacent central interfaces on the left blend into one slightly broader line. Once again, it is the optical path length that is measured, and so positions of identical surfaces in either half of the image appear displaced. The noise was markedly reduced by the removal of the mirror in this measurement. However, the signal levels were also generally rather low from the $4 \%$ reflectivity surfaces. This leads to somewhat sparse data, since the visibility of interference fringes produced by different fibres in the bundle is variable. At low signal levels, some fibres do not modulate well enough to provide a distinguishable signal. Therefore, to improve the visual appearance of the image, filtering has been applied, including a $3 \times 3$ averaging filter and additional horizontal 
averaging across 7 pixels. Spatial resolution will be only slightly reduced by the application of this level of filtering. If a higher power source were available, less filtering would be necessary to obtain a satisfactory image.

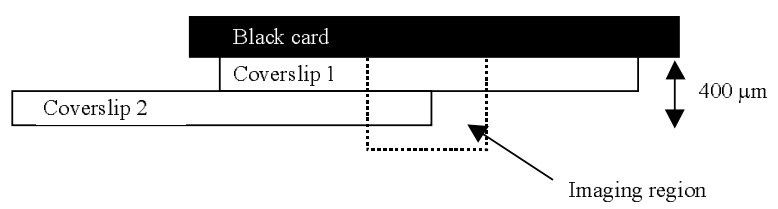

(a)

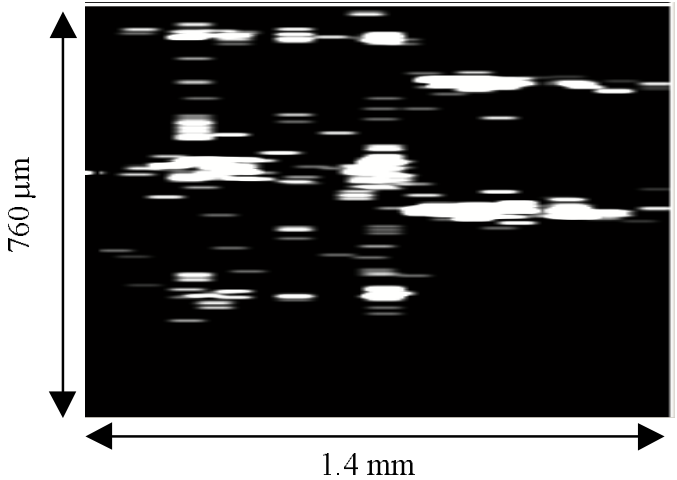

(b)

Figure 5. (a) Representation of sample composed from two stacked glass coverslips. (b) Depth slice OCT image of the central region of sample in (a).

\section{DISCUSSION}

The bundle-based approach to 3D OCT image acquisition has been successfully demonstrated above for laboratory samples of relatively high reflectivity. The elimination of $x-y$ scanning allows a completely passsive endoscopic probe to be configured.

The signal-to-noise ratio (SNR) of the images obtained is adequate for the measurements made here, but requires improvement to allow more complex samples to be studied. One straightforward way to achieve this would be by increasing the power of the source, which currently provides a maximum of only around $7 \mathrm{~mW}$ of optical power. The optical arrangement shown is not optimised for efficiency. Half the light is currently lost in the processing interferometer and a further $50 \%$ each time light passes through the beam splitter at the input of the fibre bundle. The use of polarising components and waveplates, or even a bulk-optic optical circulator, would greatly improve the optical efficiency.

There are two important questions to be addressed. The first is whether the technique has potential for measurements in biological tissue, where reflectivity values are expected to be up to two orders of magnitude lower than those of the current non-biological laboratory samples. The other issue is acquisition speed; can a bundle-based system be expected to run at speeds comparable to current systems incorporating fast $x-y$ galvo-scanners?

\subsection{SNR performance}

The SNR is dependent upon the same parameters that are relevant in a single-point OCT system. Receiver noise, excess noise and shot noise all contribute to the overall noise term? Shot noise is not usually the dominant factor in OCT systems. Excess noise is proportional to the detection bandwidth and the square of the average power at the detector. Since the current camera bandwidth is extremely low compared with the detector bandwidth for single-point systems, excess noise is also expected to be lower in the equivalent optical arrangement. An important factor limiting the performance of our current system is cross-talk between the fibres, which produces small spurious interferometric intensity oscillations for depth scan positions which are off the path-balanced condition for a particular sample interface. These arise, as discussed above, when the focused beam-waist at the sample from each fibre becomes larger than the inter-fibre spacing. Improvements to the design of the focusing optics in the probe head would reduce this problem significantly. It would also be helped by employing a bundle made up of larger, or more widely spaced fibres, which would relax the focusing requirements at the expense of a slight reduction in spatial resolution in the $x-y$ plane.

The effect of camera digitisation must also be taken into account when considering system performance. The camera currently in use digitises to 12 bits, corresponding to 4000 available signal levels. A drawback of camera detection is that the dc signal intensity cannot be filtered off before digitisation takes place. The camera therefore makes relatively 
poor use of the available digitisation range, since the interferometric signal containing the structural information occupies only about 8 bits of the total range. This limits the SNR to a maximum of around $20 \mathrm{~dB}$, which is insufficient for OCT measurements in tissue. It would therefore appear at first sight that the potential of system for biological measurements is very limited. However, there are ways in which the SNR performance could be significantly improved. One possibility is simply to choose a different camera. Although their dark current is relatively high, cameras using CMOS technology can offer a much higher dynamic range than is available from CCDs, with logarithmic compression producing SNR values up to around $60 \mathrm{~dB}$. This type of camera can also have a fast readout rate, up to hundreds of frames per second, and offers additional advantages such as the ability to access sub-areas of the chip.

Alternatively, the 3D OCT technique could be adapted to exploit the high-bandwidth, wide dynamic range capabilities of standard photodetectors, and to obtain the large increase in SNR offered by balanced detection, while also retaining the advantages offered by the bundle. This could be achieved by re-introducing $x-y$ galvo-scanners, but now positioning the scanners at the input to the bundle. Thus the input beam is scanned across the input face of the bundle, and the beam returning from the sample region is redirected, again via the scanning mirror pair, onto point detectors. In this way, the OCT information from each fibre in turn is time-multiplexed onto the detector, but the endoscopic section of the probe remains passive.

\subsection{Acquisition speed}

The processing time currently required in our system to generate a 2D image in either the $x-y$ or $x-z$ plane is of the order of several minutes. However, this is limited primarily by a slow processing algorithm. The camera is capable of reading out data at 30 frames per second; faster if pixel binning is employed to reduce the amount of data per frame. Thus, with sufficient data storage available, a set of say 100 images acquired at a sampling interval of $2 \mu \mathrm{m}$ could be obtained within $3 \mathrm{~s}$ or less and stored straight to RAM. Some further time would need to be allocated for post-processing, but a 3D OCT image would be obtained in seconds rather than minutes. Current fast systems using galvanometer scanners produce an image in around $1-5 \mathrm{~s}$, so the potential acquisition speed of the camera system is comparable with that of mechanically scanned instruments.

If the bundle-based configuration were modified to operate with galvo-scanners at the bundle input, the acquisition speed would be expected to be similar to that of the conventional scanning versions with the scanning mirrors at the probe tip. Although the optical path-length scanning in the current system is achieved using a relatively slow mechanical scanning technique, the configuration is also compatible with use of a rapid-scanning Fourier delay line, which could be used to increase the rate of depth-scanning.

\section{CONCLUSIONS}

An imaging fibre bundle has been employed to construct a full-field OCT system with a passive endoscopic probe section. It has been shown that 3D structural information can be obtained from a sample without any requirement for beam-scanning in the x-y plane normal to the optical axis. The spatial resolution of the bundle-based system compares well with that of active OCT designs using galvanometer mirrors. For the current system, the spatial resolution in the $\mathrm{x}-$ y plane and the depth resolution are both about $30 \mu \mathrm{m}$.

Measurements have been successfully made on mirrored and glass surfaces in air, over scan depths of up to about 0.75 $\mathrm{mm}$. The SNR of the system is adequate to detect glass/air interfaces, but is currently insufficient to allow biologcal samples to be imaged. However, modifications to improve the SNR to the required value have been discussed, including an increase in source power, inclusion of additional optical components to produce a more efficient configuration, and the use of alternative detection techniques. Further investigation, following implementation of these improvements, will determine the ultimate quality of the 3D images acquired when bundle-based OCT is used to examine biological tissue.

\section{ACKNOWLEDGEMENTS}

Helen Ford gratefully acknowledges a Daphne Jackson Research Fellowship sponsored by the Royal Academy of Engineering. 


\section{REFERENCES}

1. Colston, B.W., Everett, M.J., DaSilva, L.B., Otis, L.L., Stroeve, P. and Nathel, H., "Imaging of hard- and soft-tissue structure in the oral cavity by optical coherence tomography", Applied Optics, 37(16), 3582-3585, 1998.

2. Drexler, W., Morgner, U., Kartner, F.X., Pitris, C., Boppart, S.A., Li, X.D., Ippen, E.P. and Fujimoto, J.G., "Optical coherence tomography for neurosurgical imaging of human intracortical melanoma", Optics Letters, 24(17), 1221$1223,1999$.

3. Lazar, R., Brunner, H. and Steiner, R., "Optical coherence tomography (OCT) of human skin with a slow-scan CCD camera", Proc. SPIE 2925, 143-151, 1996.

4. Bamford, K.J., James, S.W., Barr, H. and Tatam, R.P., "Optical low coherence tomography of bronchial tissue", Proc. SPIE 3858, 172-179, 1999.

5. Bamford, K., James, J., Barr, H. and Tatam, R.P., "Radar detection of precancerous bronchial tissue", Lasers in Medical Science, 15, 188-194, 2000.

6. Izatt, J.A., Kulkarni, M.D., Wang, H.-W., Kobayashi, K. and Sivak, M.V. Jr., "Optical coherence tomography and microscopy in gastrointestinal tissues", IEEE Journal of Selected topics in quantum electronics, 2(4), 1017-1028, 1996.

7. Rollins, A.M. and Izatt, J.A., "Optimal interferometer designs for optical coherence tomography", Optics Letters, 24(21), 1484-1486, 1999.

8. Kersey, A.D., Marrone, M.J., Dandridge, A. and Tveten, A.B., "Control scheme for polarization-induced signal fading in multiplexed optical fibre interferometric sensors", Journal of Lightwave Technology, 6(10), 1599-1609, 1988.

9. Beddows, R., James, S.W. and Tatam, R.P., "Improved performance interferometer designs for optical coherence tomography", Presentation at the $15^{\text {th }}$ International Conference on Optical Fibre Sensors (OFS-15), Portland, Oregon, 6-10 May, 2002, IEEE Cat. No: 02EX533, 527-530.

10. Ford, H.D., Beddows, R., Casaubieilh, P. and Tatam, R.P., "Comparative signal-to-noise analysis of fibre-optic based OCT systems", Journal of Modern Optics, (in press), 2005.

11. Casaubieilh, P., Ford, H.D. and Tatam, R.P., "Optical coherence tomography with a Fizeau interferometer configuration", Proc. SPIE 5858, 2005.

12. Podoleanu A. Gh., Dobre, G.M. and Jackson D.A., "En-face coherence imaging using galvanometer scanner modulation", Optics Letters, 23(3), 147-149, 1998.

13. http://www.us.schott.com/fiberoptics/english/products/imaging/index.html.

14. Chance, B. 'Photon Migration in Tissues', Plenum Press: New York, 1988.

15. Tearney G.J., Boppart, S.A., Bouma, B.E., Brezinski, M.E., Weissman, N.J., Southern, J.F. and Fujimoto, J.G., "Scanning single-mode fiber optic catheter-endoscope for optical coherence tomography", Optics Letters, 21(7), 543-545, 1996.

16. I Balboa and R P Tatam, "Source considerations for low coherence speckle interferometry" Presented at the conference Optical Inspection and Micromeasurements II, Munich, Germany, 1997. Published in EOS/SPIE Proc . 3098, 316-324, 1997. 